\title{
Amyloid biomarkers as predictors of conversion from mild cognitive impairment to Alzheimer's dementia: a comparison of methods
}

Arnd Sörensen * (D), Ganna Blazhenets, Florian Schiller, Philipp Tobias Meyer, Lars Frings and for the Alzheimer Disease Neuroimaging Initiative

\begin{abstract}
Background: Amyloid- $\beta$ (A $\beta$ ) PET is an established predictor of conversion from mild cognitive impairment (MCI) to Alzheimer's dementia (AD). We compared three PET (including an approach based on voxel-wise Cox regression) and one cerebrospinal fluid (CSF) outcome measures in their predictive power.

Methods: Datasets were retrieved from the ADNI database. In a training dataset $(N=159)$, voxel-wise Cox regression and principal component analyses were used to identify conversion-related regions (Cox-VOI and AD conversionrelated pattern (ADCRP), respectively). In a test dataset $(N=129)$, the predictive value of mean normalized ${ }^{18} \mathrm{~F}$ florbetapir uptake (SUVR) in AD-typical brain regions (composite SUVR) or the Cox-VOI and the pattern expression score (PES) of ADCRP and CSF $A \beta_{42} / A \beta_{40}$ as predictors were compared by Cox models (corrected for age and sex).

Results: All four $A \beta$ measures were significant predictors $(p<0.001)$. Prediction accuracies (Harrell's $c$ ) showed stepwise significant increases from Cox-SUVR ( $c=0.71 ; \mathrm{HR}=1.84$ per $Z$-score increase), composite SUVR ( $c=0.73 ; \mathrm{HR}=2.18)$, CSF A $\beta_{42} / A \beta_{40}(c=0.75 ; H R=3.89)$ to PES $(c=0.77 ; H R=2.71)$.

Conclusion: The PES of ADCRP is the most predictive A $\beta$ PET outcome measure, comparable to CSF $A \beta_{42} / A \beta_{40}$, with a slight but statistically significant advantage.
\end{abstract}

Keywords: Amyloid biomarkers, Mild cognitive impairment, Alzheimer's dementia, Conversion prediction, PET image evaluation

\section{Introduction}

Amyloid- $\beta$ (A $\beta$ ) PET (e.g., using ${ }^{18}$ F-florbetapir, also known as $\left.{ }^{18} \mathrm{~F}-\mathrm{AV}-45\right)$ is an established biomarker for $\mathrm{A} \beta$ pathology [1] and might hence be used to predict conversion from mild cognitive impairment $(\mathrm{MCI})$ to Alzheimer's dementia (AD) [2-5]. Earlier studies commonly used binary $A \beta$ outcome measures based on visual reads

\footnotetext{
* Correspondence: arnd.soerensen@uniklinik-freiburg.de Department of Nuclear Medicine, Medical Center - University of Freiburg, Faculty of Medicine, University of Freiburg, Hugstetter Str. 55, 79106 Freiburg, Germany
}

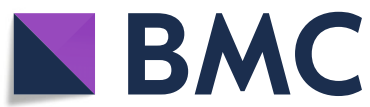

C C The Author(s). 2020 Open Access This article is licensed under a Creative Commons Attribution 4.0 International License, which permits use, sharing, adaptation, distribution and reproduction in any medium or format, as long as you give appropriate credit to the original author(s) and the source, provide a link to the Creative Commons licence, and indicate if changes were made. The images or other third party material in this article are included in the article's Creative Commons licence, unless indicated otherwise in a credit line to the material. If material is not included in the article's Creative Commons licence and your intended use is not permitted by statutory regulation or exceeds the permitted use, you will need to obtain permission directly from the copyright holder. To view a copy of this licence, visit http://creativecommons.org/licenses/by/4.0/. The Creative Commons Public Domain Dedication waiver (http://creativecommons.org/publicdomain/zero/1.0/) applies to the data made available in this article, unless otherwise stated in a credit line to the data. or volume of interest (VOI) analyses [2-4] or continuous $A \beta$ measures relying on composite anatomical regions (i.e., treating all voxels equally) [5]. However, we recently demonstrated that voxel-wise principal component analysis (PCA) provides an AD-specific covariance pattern among voxels, which is superior to the aforementioned conventional approaches [6]. Another promising prognostic method in PET data analysis is voxel-wise Cox regression, which we recently applied to ${ }^{18} \mathrm{~F}$-FDG PET in MCI [7]. However, the 
best image evaluation method is still a matter of debate.

Aside from $A \beta$ PET, the $A \beta$ concentration in cerebrospinal fluid (CSF) is also an established non-imaging biomarker for $A \beta$ pathology [8]. The ratio of the concentration of $A \beta_{42}$ to the concentration of $A \beta_{40}\left(A \beta_{42} / A \beta_{40}\right)$ showed the best diagnostic performance among different evaluation methods $[9,10]$.

While many studies consider CSF $A \beta$ and $A \beta$ PET to be equally capable of predicting cognitive decline [11-16], some find a slight advantage for A $\beta$ PET [17] in this regard. On the other hand, recent findings suggest that CSF indicates abnormal $A \beta$ accumulation before $A \beta$ PET in the earliest stages of the disease [18] and that $A \beta$ PET is more strongly connected to disease progression [19].

Against this background, we used a large dataset from the Alzheimer's disease neuroimaging initiative (ADNI) to compare the aforementioned three continuous $A \beta$ PET outcome measures and the $A \beta_{42} / A \beta_{40}$ ratio in CSF in their ability to predict conversion from MCI to $\mathrm{AD}$. We used a training dataset, to which voxel-wise Cox regression and PCA were applied to identify conversionrelated regions, and a test dataset, by use of which all four methods were prospectively compared.

\section{Material and methods \\ Subjects}

All data used in the present study was provided by the ADNI database (ClinicalTrials.gov Identifier: NCT00106899), and comprehensive information about the ADNI project can be found at the official website (www.adni-info.org).

For our previous study [6], $319{ }^{18} \mathrm{~F}-\mathrm{AV}-45$ PET scans were retrieved from the ADNI database. Patients with the following criteria were included: MCI diagnosis ("DX-Score" 2, suspected incipient Alzheimer disease with subjective and objective memory deficits) and a baseline ${ }^{18} \mathrm{~F}-\mathrm{AV}-45$ scan, at least 25 points on MiniMental State Examination (MMSE), follow-up time of at least 6 months, and no bidirectional change of diagnosis (MCI to AD and back). This dataset was randomly split into two equally sized cohorts: a training and a test dataset. The training dataset has been used for voxel-wise Cox regression and PCA to identity the Cox-VOI in the present study (see below) and the ADCRP in our earlier study [6]. The test dataset for the present study was further reduced to a subset of 129 patients with available data on amyloid- $\beta$ concentration in the CSF. Details on clinical and demographic characteristics can be found in Table 1.

\section{${ }^{18} \mathrm{~F}-\mathrm{AV}$-45 PET data and image preprocessing}

Four 5-min frames were used (50 to $70 \mathrm{~min}$ after injection of $\left.{ }^{18} \mathrm{~F}-\mathrm{AV}-45\right)$. A motion correction was applied if necessary. All frames were summed into a single image dataset. After spatial normalization to an in-house template $\left({ }^{18} \mathrm{~F}\right.$-Florbetapir, constructed from nine $\mathrm{A} \beta$-positive and seven $A \beta$-negative elderly normal controls) in $\mathrm{MNI}$ space, spatial smoothing with a 12-mm FWHM isotropic Gaussian kernel was applied. Full details on PET acquisition protocols can be found on the ADNI website.

\section{PET image analysis: training dataset}

For each voxel, independently, a Cox model was fitted with the $z$-scaled SUVR as a predictor variable, adjusted for age and sex. Among those voxels that showed a significant association between SUVR and conversion from MCI to AD (FDR-corrected, $p<0.01$ ), the $20 \%$ of voxels with the highest hazard ratios (HR) were combined into the "Cox-VOI."

The same training dataset was used in our previous study [6] to identify the AD conversion-related pattern (ADCRP), which was also used in the present study for the evaluation of the test dataset.

\section{Cox regressions: test dataset}

In the test dataset, four Cox models were built in order to compare their prognostic performance concerning MCI-to-AD conversion. Each model included one of the four $A \beta$ measures as the main predictor variable (all $z$ scaled), as well as age and sex as covariates:

Table 1 Clinical and demographic characteristics of the included Alzheimer's Disease Neuroimaging Initiative (ADNI) participants

\begin{tabular}{|c|c|c|c|c|}
\hline & \multicolumn{2}{|c|}{ Training dataset $(n=159)$} & \multicolumn{2}{|c|}{ Test dataset $(n=129)$} \\
\hline & $\mathrm{MCl}-\mathrm{c}(n=41)$ & MCl-nc $(n=118)$ & $\mathrm{MCl}-\mathrm{c}(n=29)$ & MCI-nc $(n=101)$ \\
\hline Mean age $( \pm$ S.D.) [years] & $72 \pm 7$ & $73 \pm 8$ & $73 \pm 7$ & $73 \pm 8$ \\
\hline $\operatorname{Sex}[\mathrm{m} / \mathrm{f}]$ & $14 / 27$ & $55 / 63$ & $15 / 14$ & $59 / 42$ \\
\hline Mean $A \beta_{42} / A \beta_{40}$ in CSF $( \pm$ S.D. $)$ & Not assessed & & $0.10 \pm 0.06$ & $0.15 \pm 0.06$ \\
\hline PES of ADCRP $( \pm$ S.D.) & $18 \pm 19$ & $-6 \pm 18$ & $16 \pm 13$ & $-2 \pm 17$ \\
\hline Median follow-up time (95\% C.I.) [months] & $48(36-51)$ & & $47(35-51)$ & \\
\hline Cox-SUVR ( \pm S.D.) & $1.7 \pm 0.3$ & $1.4 \pm 0.2$ & $1.6 \pm 0.2$ & $1.5 \pm 0.2$ \\
\hline Composite SUVR ( \pm S.D.) & Not assessed & & $1.6 \pm 0.2$ & $1.4 \pm 0.2$ \\
\hline
\end{tabular}

MCI-c mild cognitive impairment-converters, MCI-nc MCI non-converters, S.D. standard deviation, CSF cerebrospinal fluid, PES pattern expression score, SUVR standardized uptake value ratio 
1. Composite SUVR: the mean standardized uptake value ratio (SUVR, reference region: cerebellum) was calculated within a VOI comprising anatomical regions with the highest $\mathrm{A} \beta$ load in $\mathrm{AD}$ (established in a previous study [20] using Pittsburgh compound B).

2. Cox-SUVR: the mean SUVR within the Cox-VOI was read-out and weighted in a voxel-wise fashion by its HR (calculated in the training dataset; thus, voxel with a higher predictive value contributed more).

3. PES of ADCRP: the individual pattern expression score (PES) was calculated for the ADCRP that has been established in our previous study [6]. The PES was evaluated by the topographic profile rating algorithm, as described in [21].

4. CSF $A \beta_{42} / A \beta_{40}$ : the $A \beta_{42} / A \beta_{40}$ concentration ratio from CSF was used the main predictor variable.

\section{Results}

\section{Training dataset}

Figure 1 depicts three-dimensional surface projections of HRs calculated by voxel-wise Cox regression in the training dataset, which follows the known distribution of $A \beta$ pathology in AD. Voxels with the top $20 \%$ of HR are illustrated in Fig. 2 (red regions, $79 \mathrm{ml}$ ), which cover parts of the striatum and mesial frontal and superior temporal cortices as well as the precuneus and insula. These regions only partially overlap $(42 \mathrm{ml})$ with the larger anatomical VOI used to calculate the composite SUVR (Fig. 2, blue regions, $584 \mathrm{ml}$ ).

\section{Test dataset}

All four Cox models significantly predicted MCI-to-AD conversion in the test dataset (all Wald tests $p<0.001$ ).
Pairwise comparisons between models (Fig. 3), using the likelihood ratio test, revealed significant step-wise improvements $(p<0.001)$ from the model with Cox-SUVR $(\mathrm{HR}=1.84$ per $Z$-score increase [95\% C.I. 1.31-2.56] $)$ with a concordance of Harrell's $c=0.71$ (95\% C.I. 0.59$0.82)$ to the model incorporating composite SUVR (HR = 2.18 [1.51-3.16]) with $c=0.73(0.62-0.84)$, to the model relying on CSF $A \beta_{42} / A \beta_{40}(H R=3.89$ [2.10-7.19]) with $c=0.75(0.65-0.87)$, and, finally, to the model using PES of ADCRP as a predictor (HR of 2.71 [1.78-4.13]) with $c=0.77(0.66-0.89)$.

\section{Discussion}

In the present study, all three tested $A \beta$ PET outcome measures and the CSF $A \beta_{42} / A \beta_{40}$ ratio were able to predict the development of $\mathrm{AD}$ in patients diagnosed with MCI. Among these Cox models, each containing the main predictor variable and all corrected for age and sex, the model with the PES of ADCRP showed the highest concordance (Harrell's c). Binary outcome measures such as visual reads or threshold-based methods have been neglected for this study, as we have shown in our previous work [6] that binary measures perform worse than continuous $A \beta$ PET outcome measures.

Cox-SUVR represents a novel A $\beta$ PET outcome measure, which we explored based on our previous observation that voxel-wise Cox regression is a promising predictor of MCI-to-AD conversion when applied to FDG PET data [7]. In the training dataset, we tested several HR thresholds (using the top 50\%, 30\%, 20\%, and $10 \%$ ) for Cox-SUVR calculation. All threshold-based approaches showed improvement over using simply all significant voxels. The $20 \%$ threshold performed best and

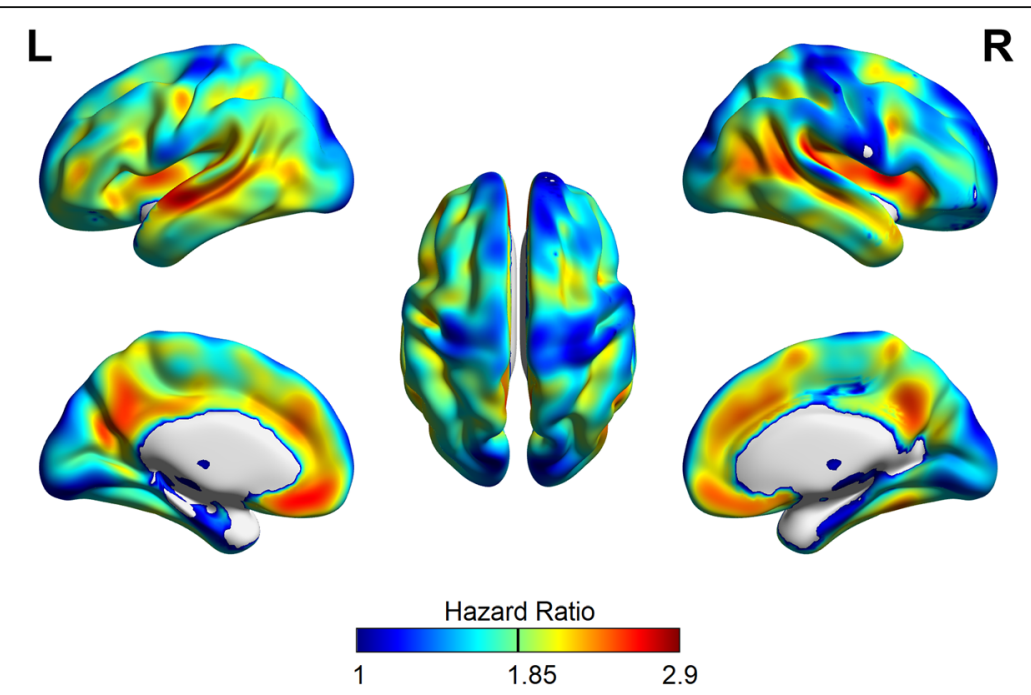

Fig. 1 Surface projections of the hazard ratios (HRs) from the voxel-wise Cox regressions in the training dataset. HR is expressed per one unit increase of the $z$-scaled standardized uptake value ratio (SUVR; reference region: cerebellum) of ${ }^{18} \mathrm{~F}$-florbetapir 


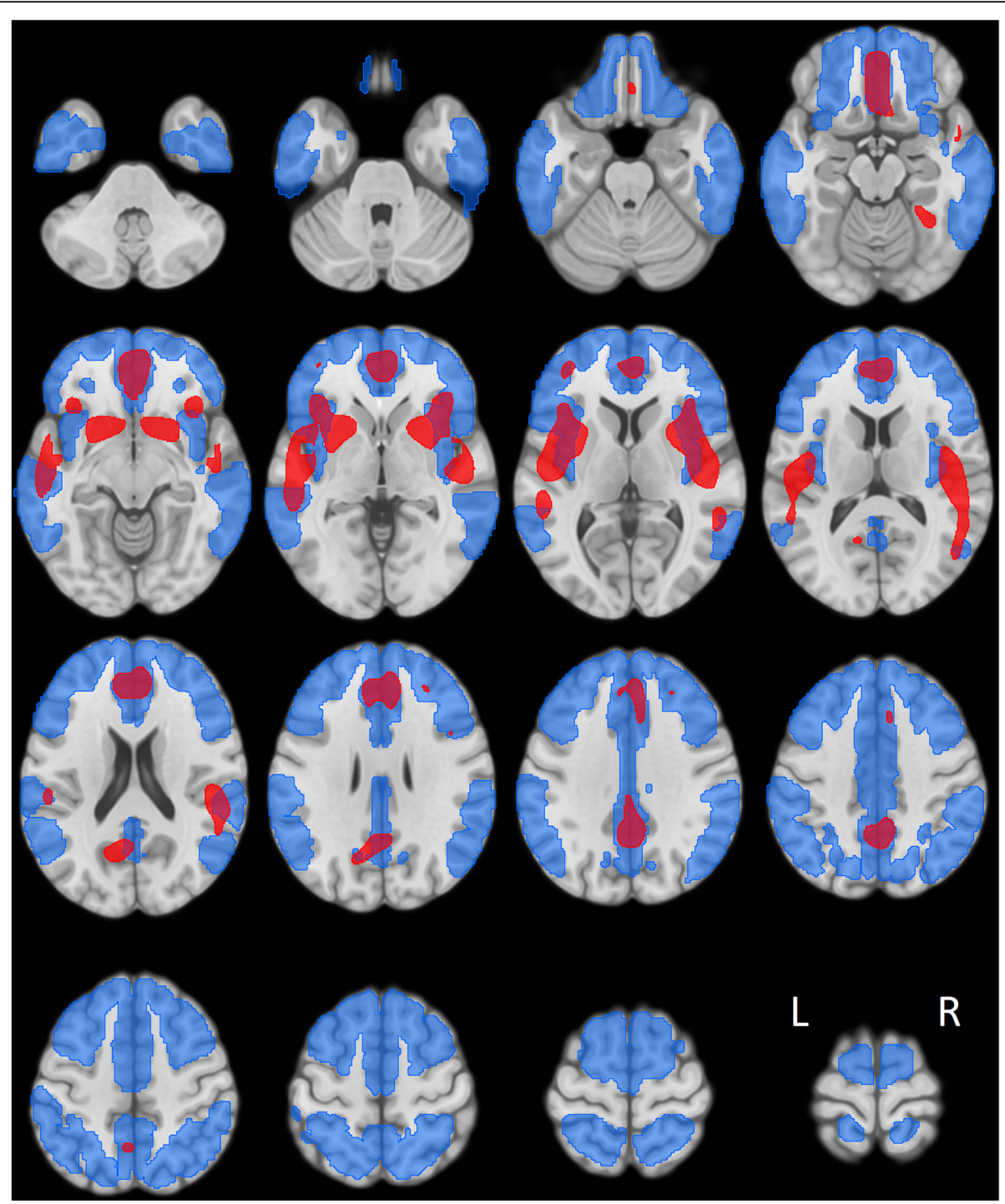

Fig. 2 Volume of interest (VOI) overlays (onto the MNI-152 MRI template) showing significant voxels with top 20\% hazard ratios (HRs) from voxelwise Cox regressions in the training dataset (red, Cox-VOI) used to assess Cox-SUVR and the anatomical VOI (blue; taken from [20]) employed for composite SUVR calculation

was thus chosen. The distribution and magnitude of voxel-wise HRs (Fig. 1) are largely in agreement with regions of known $\mathrm{A} \beta$ deposition in $\mathrm{AD}$ [22-25], which were used for the calculation of composite SUVR as an established prognostic marker [20]. However, the CoxVOI $(79 \mathrm{ml})$ was much smaller than the anatomical VOI $(584 \mathrm{ml})$, with only little overlap $(42 \mathrm{ml})$.

Both $A \beta$ PET and determination of $A \beta$ from CSF offer an excellent way to predict the development of Alzheimer's disease: $A \beta$ from CSF detects amyloid pathology earlier than A $\beta$ PET [18], but both are predictors in their own right, such that patients with concordant CSF and amyloid PET findings have a worse prognosis than those with discordant findings $[26,27]$. The choice regarding which of the two will be applied should depend on the availability and the patient's preference.

Evaluation of $A \beta$ PET by PES from PCA might easily be implemented to support clinical routine since Eidelberg [28] developed a freely available toolbox (Scanvp/ SSMPCA toolbox available at the website of the Feinstein Institute for Medical Research, http://feinsteinneuroscience.org/software, [29]) for Statistical Parametric Mapping (SPM). The prognostic value of A $\beta$ PET might also benefit from scanner development (higher spatial resolution and better signal-to-noise ratio). Finally, an ideal combination in the future of $\mathrm{AD}$ diagnosis might be that of $\mathrm{A} \beta$ PET with tau PET, allowing for a classification (regarding " $\mathrm{A}$ " and " $\mathrm{T}$ ") following the NIA-AA research framework. 


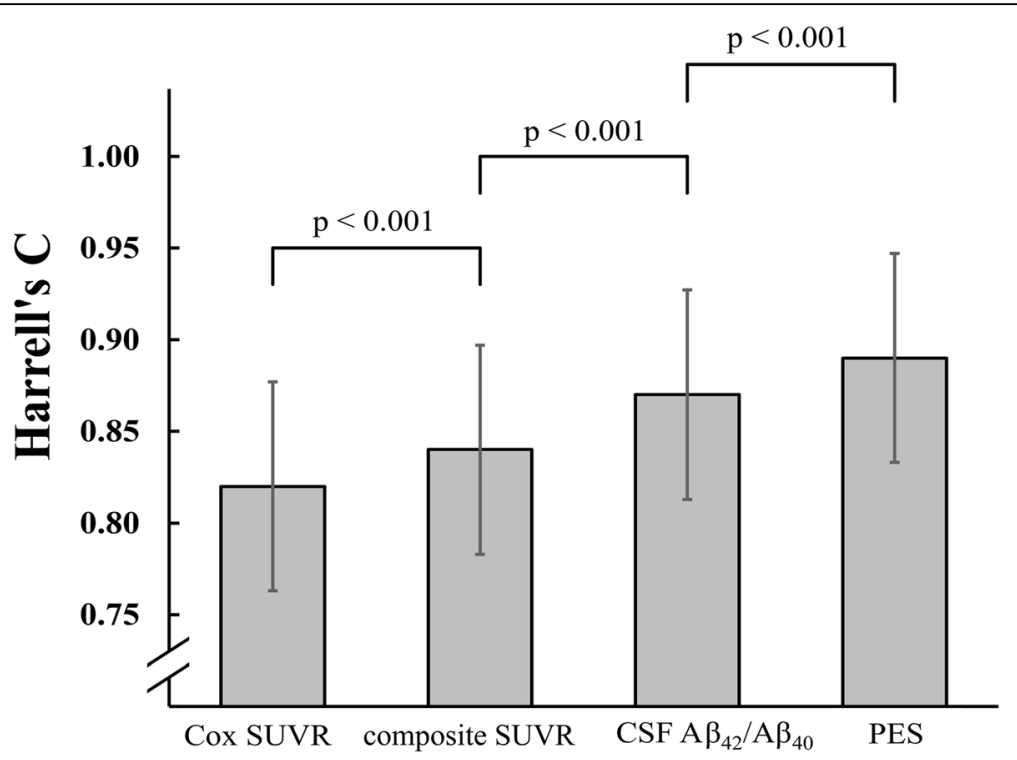

Fig. 3 Prediction concordance (Harrell's $c$ ) and respective confidence intervals of the four Cox regression models (corrected for age and sex). $P$ values were derived from pair-wise likelihood ratio tests

\section{Limitations}

In contrast to the novel Cox-SUVR, composite SUVR and the CSF $A \beta_{42} / A \beta_{40}$ ratio are established diagnostic and prognostic biomarkers of AD [1-5, 8]. Several other factors might be considered to contribute to the performance ranking obtained from our analyses: First, only for the derivation of the Cox-VOI the time-to-conversion information was used, but unexpectedly, it performed worst in the overall comparison. We assume, however, that time information might prove more beneficial in datasets with a larger inter-individual variability of time-to-conversion (the interquartile range was just 13 months in the test dataset). Secondly, while CSF $A \beta_{42} / A \beta_{40}$ is an integral measure for the production and clearance of $A \beta$ at a given time and across all brain regions, $A \beta$ PET represents a direct measurement of spatial $A \beta$ accumulation. Thirdly, while all PET measures tested here provide regional weighting, this is only binary in the case of composite SUVR. By contrast, regional weighting is continuous in Cox-SUVR and PES. Last, the PES calculation includes not only regions with high $A \beta$ accumulation or associated with an increased risk of conversion, but all voxels of the brain (i.e., possibly also patterns associated with lower risk or protective features). A combination of these factors might explain why the PES of ADCRP performed best in this comparison-by a small margin.

\section{Conclusion}

All tested $A \beta$ outcome measures significantly predicted conversion from MCI to AD. The PES of ADCRP is comparable to CSF $A \beta_{42} / A \beta_{40}$, with a slight but statistically significant advantage over CSF $A \beta_{42} / A \beta_{40}$.

\section{Abbreviations}

$A \beta$ : Amyloid- $\beta_{;} A \beta_{42} / A \beta_{40}$ : Ratio of the concentration of $A \beta_{42}$ to the concentration of $A \beta_{40}$; $A D$ : Alzheimer's dementia; ADCRP: AD conversionrelated pattern; ADNI: Alzheimer's Disease Neuroimaging Initiative; CSF: Cerebrospinal fluid; ${ }^{18} \mathrm{~F}-\mathrm{AV}-45:{ }^{18} \mathrm{~F}$-florbetapir; FDR: False discovery rate; FWHM: Full width at half maximum; HR: Hazard ratio; MCl: Mild cognitive impairment; MMSE: Mini-Mental State Examination; MNI: Montreal Neurological Institute; PCA: Principal component analysis; PES: Pattern expression score; PET: Positron emission tomography; SPM: Statistical parametric mapping; SUVR: Standardized uptake value ratio; VOI: Volume of interest

\section{Acknowledgements}

The use in this work of the ScAnVP software, copyright@ 2020 The Feinstein Institute for Medical Research, is hereby acknowledged. Data used in the preparation of this article were obtained from the Alzheimer's Disease Neuroimaging Initiative (ADNI) database (adni.loni.usc.edu). As such, the investigators within the ADNI contributed to the design and implementation of $\mathrm{ADNI}$ and/or provided data but did not participate in the analysis or writing of this report. A complete listing of ADNI investigators can be found at http://adni.loni.usc.edu/wp-content/uploads/how_to_apply/ADNI_ Acknowledgement_List.pdf.

\section{Authors' contributions}

All authors read and approved the final manuscript.

\section{Funding}

Not applicable. Open Access funding enabled and organized by Projekt DEAL.

\section{Availability of data and materials}

After registration, the initial imaging and patient metadata are available from the official ADNI website.

\section{Ethics approval and consent to participate}

All procedures performed in studies involving human participants were in accordance with the ethical standards of the institutional and/or national research committee and with the 1964 Helsinki Declaration and its later amendments or comparable ethical standards.

Consent for publication

All patients provided written informed consent, see ADNI. 


\section{Competing interests}

Dr. Meyer reports personal fees from GE and personal fees from Philips, outside the submitted work. All other authors have nothing to disclose.

Received: 17 September 2020 Accepted: 5 November 2020

Published online: 19 November 2020

\section{References}

1. Jack CR, Bennett DA, Blennow K, Carrillo MC, Dunn B, Haeberlein SB, et al. NIA-AA Research Framework: toward a biological definition of Alzheimer's disease. Alzheimers Dement. 2018;14(4):535-62 Available from: https://doi. org/10.1016/j.jalz.2018.02.018.

2. Schreiber S, Landau SM, Fero A, Schreiber F, Jagust WJ. Comparison of visual and quantitative florbetapir $F 18$ positron emission tomography analysis in predicting mild cognitive impairment outcomes. JAMA Neurol. 2015;72(10): 1183 Available from: https://doi.org/10.1001/jamaneurol.2015.1633.

3. Grimmer T, Wutz C, Alexopoulos P, Drzezga A, Forster S, Forstl H, et al. Visual versus fully automated analyses of 18F-FDG and amyloid PET for prediction of dementia due to Alzheimer disease in mild cognitive impairment. J Nucl Med. 2016;57(2):204-7.

4. Frings L, Hellwig S, Bormann T, Spehl TS, Buchert R, Meyer PT. Amyloid load but not regional glucose metabolism predicts conversion to Alzheimer's dementia in a memory clinic population. Eur J Nucl Med Mol Imaging 2018; Available from: http://www.ncbi.nlm.nih.gov/pubmed/29546632\%0A; http://link.springer.com/10.1007/s00259-018-3983-6.

5. Trzepacz PT, Yu P, Sun J, Schuh K, Case M, Witte MM, et al. Comparison of neuroimaging modalities for the prediction of conversion from mild cognitive impairment to Alzheimer's dementia. Neurobiol Aging. 2014;35(1): 143-51 Available from: http://linkinghub.elsevier.com/retrieve/pii/S019745 8013002790 .

6. Blazhenets G, Ma Y, Sörensen A, Schiller F, Rücker G, Eidelberg D, et al. Predictive value of 18 F-florbetapir and 18 F-FDG PET for conversion from mild cognitive impairment to Alzheimer dementia. J Nucl Med. 2019; jnumed.119.230797. Available from: http://jnm.snmjournals.org/lookup/doi/1 0.2967/jnumed.119.230797.

7. Sörensen A, Blazhenets G, Rücker G, Schiller F, Meyer PT, Frings L. Prognosis of conversion of mild cognitive impairment to Alzheimer's dementia by voxel-wise Cox regression based on FDG PET data. Neurolmage Clin. 2018; 101,637. [cited 2018 Dec 11]Available from: https://www.sciencedirect.com/ science/article/pii/S2213158218303851?via\%3Dihub.

8. Andreasen N, Minthon L, Davidsson P, Vanmechelen E, Vanderstichele $H$, Winblad B, et al. Evaluation of CSF-tau and CSF-Abeta42 as diagnostic markers for Alzheimer disease in clinical practice. Arch Neurol. 2001;58(3): 373-9. [cited 2020 Jan 16] Available from: http://archneur.jamanetwork.com/ article.aspx?doi=10.1001/archneur.58.3.373.

9. Nutu M, Zetterberg $H$, Londos E, Minthon L, Nägga K, Blennow K, et al. Evaluation of the cerebrospinal fluid amyloid- $\beta 1-42$ /amyloid- $\beta 1-40$ ratio measured by alpha-LISA to distinguish Alzheimer's disease from other dementia disorders. Dement Geriatr Cogn Disord. 2013;36(1-2):99-110 [cited 2020 Jan 16]Available from: https://www.karger.com/Article/ FullText/353442.

10. Lewczuk P, Lelental N, Spitzer P, Maler JM, Kornhuber J. Amyloid- $\beta$ 42/40 cerebrospinal fluid concentration ratio in the diagnostics of Alzheimer's disease: validation of two novel assays. J Alzheimer's Dis. 2014;43(1):183-91. [cited 2020 Jan 16] Available from: https://www.medra.org/servlet/ aliasResolver?alias=iospress\&doi=10.3233/JAD-140771.

11. Schipke CG, Koglin N, Bullich S, Joachim LK, Haas B, Seibyl J, et al. Correlation of florbetaben PET imaging and the amyloid peptide Aß42 in cerebrospinal fluid. Psychiatry Res Neuroimaging. 2017;265(May 2016):98101 Available from: https://doi.org/10.1016/j.pscychresns.2016.10.011.

12. Palmqvist $\mathrm{S}$, Zetterberg $\mathrm{H}$, Mattsson $\mathrm{N}$, Johansson $\mathrm{P}$, Minthon $\mathrm{L}$, Blennow $\mathrm{K}$, et al. Detailed comparison of amyloid PET and CSF biomarkers for identifying early Alzheimer disease. Neurology. 2015;85(14):1240-9 [cited 2020 Feb 7] Available from: http://www.neurology.org/lookup/doi/10.1212/ WNL.0000000000001991.

13. Leuzy A, Chiotis K, Hasselbalch SG, Rinne JO, de Mendonça A, Otto M, et al. Pittsburgh compound B imaging and cerebrospinal fluid amyloid- $\beta$ in a multicentre European memory clinic study. Brain. 2016;139(9):2540-53 Available from: https:/academic.oup.com/brain/article-lookup/doi/10.1093/ brain/aww160.
14. Fagan AM, Mintun MA, Mach RH, Lee S-Y, Dence CS, Shah AR, et al. Inverse relation between in vivo amyloid imaging load and cerebrospinal fluid $A \beta$ 42 in humans. Ann Neurol. 2006;59(3):512-9. [cited 2020 Feb 7] Available from: http://doi.wiley.com/10.1002/ana.20730.

15. Landau SM, Lu M, Joshi AD, Pontecorvo M, Mintun MA, Trojanowski JQ, et al. Comparing positron emission tomography imaging and cerebrospinal fluid measurements of $\beta$-amyloid. Ann Neurol. 2013;74(6):826-36 Available from: http://doi.wiley.com/10.1002/ana.23908.

16. Hansson O, Seibyl J, Stomrud E, Zetterberg H, Trojanowski JQ, Bittner T, et al. CSF biomarkers of Alzheimer's disease concord with amyloid- $\beta$ PET and predict clinical progression: a study of fully automated immunoassays in BioFINDER and ADNI cohorts. Alzheimer's Dement. 2018;14(11):1470-81. [cited 2020 Feb 28] Available from: http://doi.wiley.com/10.1016/j.jalz.2018.01.010.

17. Ramusino MC, Garibotto V, Bacchin R, Altomare D, Dodich A, Assal F, et al. Incremental value of amyloid-PET versus CSF in the diagnosis of Alzheimer's disease. Eur J Nucl Med Mol Imaging. 2020;47(2):270-80 [cited 2020 Feb 7] Available from: http://link.springer.com/10.1007/s00259-019-04466-6.

18. Palmqvist S, Mattsson N, Hansson O. Cerebrospinal fluid analysis detects cerebral amyloid- $\beta$ accumulation earlier than positron emission tomography. Brain. 2016;139(4):1226-36 Available from: https://academic. oup.com/brain/article-lookup/doi/10.1093/brain/aww015.

19. Mattsson N, Insel PS, Donohue M, Landau S, Jagust WJ, Shaw LM, et al. Independent information from cerebrospinal fluid amyloid- $\beta$ and florbetapir imaging in Alzheimer's disease. Brain. 2015;138(3):772-83 [cited 2020 Feb 7] Available from: https://academic.oup.com/brain/article-lookup/doi/10.1093/ brain/awu367.

20. Frings L, Hellwig S, Spehl TS, Bormann T, Buchert R, Vach W, et al. Asymmetries of amyloid- $\beta$ burden and neuronal dysfunction are positively correlated in Alzheimer's disease. Brain. 2015;138(10):3089-99 Available from: https://academic.oup.com/brain/article-lookup/doi/10.1093/brain/ awv229.

21. Spetsieris $P G$, Eidelberg D. Scaled subprofile modeling of resting state imaging data in Parkinson's disease: methodological issues. Neuroimage 2011;54(4):2899-914 [cited 2020 Jan 28]Available from: https://linkinghub. elsevier.com/retrieve/pii/S1053811910013170.

22. Jack CR, Barrio JR, Kepe V. Cerebral amyloid PET imaging in Alzheimer's disease. Acta Neuropathol. 2013;126(5):643-57. [cited 2020 Feb 5] Available from: http://link.springer.com/10.1007/s00401-013-1185-7.

23. Villain $N$, Chételat $G$, Grassiot $B$, Bourgeat $P$, Jones $G$, Ellis KA, et al. Regional dynamics of amyloid- $\beta$ deposition in healthy elderly, mild cognitive impairment and Alzheimer's disease: a voxelwise PiB-PET longitudinal study. Brain. 2012;135(7):2126-39 Available from: https://doi.org/10.1093/brain/ aws 125.

24. Villemagne $V L$, Pike $K E$, Chételat $G$, Ellis KA, Mulligan RS, Bourgeat $P$, et al. Longitudinal assessment of $A \beta$ and cognition in aging and Alzheimer disease. Ann Neurol. 2011;69(1):181-92. Available from: https://doi.org/10. 1002/ana.22248

25. Rinne JO, Brooks DJ, Rossor MN, Fox NC, Bullock R, Klunk WE, et al. 11C-PiB PET assessment of change in fibrillar amyloid- $\beta$ load in patients with Alzheimer's disease treated with bapineuzumab: a phase 2, double-blind, placebo-controlled, ascending-dose study. Lancet Neurol. 2010;9(4):363-72 Available from: https://doi.org/10.1016/S1474-4422(10)70043-0.

26. de Wilde A, Reimand J, Teunissen CE, Zwan M, Windhorst AD, Boellaard R, et al. Discordant amyloid- $\beta$ PET and CSF biomarkers and its clinical consequences. Alzheimers Res Ther. 2019;11(1):78 Available from: https:// pubmed.ncbi.nlm.nih.gov/31511058.

27. Reimand J, Collij L, Scheltens P, Bouwman F, Ossenkoppele R. Association of amyloid- $\beta$ CSF/PET discordance and tau load five years later. Neurology. 2020. https://doi.org/10.1212/WNL.0000000000010739 Available from: http:// www.neurology.org/lookup/doi/10.1212/WNL.0000000000010739.

28. Eidelberg D. Metabolic brain networks in neurodegenerative disorders: a functional imaging approach. Trends Neurosci. 2009/09/16. 2009;32(10):54857. Available from: https://www.ncbi.nlm.nih.gov/pmc/articles/PMC2782537/.

29. Spetsieris P, Ma Y, Peng S, Ko JH, Dhawan V, Tang CC, et al. Identification of disease-related spatial covariance patterns using neuroimaging data. J Vis Exp. 2013;(76). [cited 2020 Jan 28] Available from: http://www.jove.com/ video/50319/identification-disease-related-spatial-covariance-patterns-using.

\section{Publisher's Note}

Springer Nature remains neutral with regard to jurisdictional claims in published maps and institutional affiliations. 